\title{
The Compact Central Object in the Supernova Remnant G266.2-1.2
}

\author{
Oleg Kargaltsev, George G. Pavlov, Divas Sanwal, and Gordon P. Garmire \\ Dept. of Astronomy and Astrophysics, The Pennsylvania State University, 525 Davey Lab, \\ University Park, PA 16802 \\ green, pavlov, divas, garmire@astro.psu.edu
}

\begin{abstract}
We observed the compact central object CXOU J085201.4-461753 in the supernova remnant G266.2-1.2 (RX J0852.0-4622) with the Chandra ACIS detector in timing mode. The spectrum of this object can be described by a blackbody model with the temperature $k T=404 \pm 5 \mathrm{eV}$ and radius of the emitting region $R=0.28 \pm 0.01 \mathrm{~km}$, at a distance of $1 \mathrm{kpc}$. Power-law and thermal plasma models do not fit the source spectrum. The spectrum shows a marginally significant feature at $1.68 \mathrm{keV}$. Search for periodicity yields two candidate periods, about 301 $\mathrm{ms}$ and $33 \mathrm{~ms}$, both significant at a $2.1 \sigma$ level; the corresponding pulsed fractions are $13 \%$ and $9 \%$, respectively. We find no evidence for long-term variability of the source flux, nor do we find extended emission around the central object. We suggest that CXOU J085201.4-461753 is similar to CXOU J232327.9+584842, the central source of the supernova remnant Cas A. It could be either a neutron star with a low or regular magnetic field, slowly accreting from a fossil disk, or, more likely, an isolated neutron star with a superstrong magnetic field. In either case, a conservative upper limit on surface temperature of a $10 \mathrm{~km}$ radius neutron star is about $90 \mathrm{eV}$, which suggests accelerated cooling for a reasonable age of a few thousand years.
\end{abstract}

Subject headings: stars: neutron — supernova remnants: individual (G266.2-1.2) - X-rays: individual (CXOU J085201.4-461753)

\section{Introduction}

The shell-like supernova remnant (SNR) G266.2-1.2 (also known as RX J0852.0-4622, or "Vela Junior") at the south-east corner of the Vela SNR was discovered by Aschenbach 
(1998) in the ROSAT All-Sky Survey data. Possible detection of the $1.156 \mathrm{MeV} \gamma$-ray line of the radioactive isotope ${ }^{44} \mathrm{Ti}$ (half-life $\sim 90 \mathrm{yr}$ ) with the Compton Gamma-Ray Observatory (Iyudin et al. 1998) may imply a very young SNR age of $\sim 680 \mathrm{yr}$, at a distance of $\sim 200$ pc. Aschenbach, Iyudin, \& Schönfelder (1999) estimated upper limits of $1100 \mathrm{yr}$ for the age, and $500 \mathrm{pc}$ for the distance. Observations with ASCA (Tsunemi et al. 2000; Slane et al. 2001) demonstrate that the X-ray spectra of the SNR shell are nonthermal. Fits of these spectra with a power-law (PL) model yield a hydrogen column density substantially higher than that for the Vela SNR, implying a plausible distance to the remnant of 1-2 kpc, and an age of a few thousand years.

Aschenbach (1998) suggests that G266.2-1.2 was created by a core-collapse supernova that left a compact remnant - a neutron star (NS) or a black hole (BH). Three compact remnant candidates have been reported from the observations with ROSAT (Aschenbach 1998; Aschenbach et al. 1999), ASCA (Slane et al. 2001), and Beppo-SAX (Mereghetti 2001). Pavlov et al. (2001) observed G266.2-1.2 with the Chandra Advanced CCD Imaging Spectrometer (ACIS) and found only one bright X-ray source, CXOU J085201.4-461753 (J0852 hereafter), close to the SNR center. They measured the source position with accuracy better than $2^{\prime \prime}$ and proved that J0852 is not an X-ray counterpart of bright optical stars in the field. Follow-up optical observations (Pavlov et al. 2001; Mereghetti, Pelizzoni, \& De Luca 2002a) revealed an object located only 2".4 south-west of the J0852. The colors of the optical source are consistent with those of a main sequence star at a distance of 1.5-2.5 kpc; most likely, this is a field star. The limiting optical magnitudes at the position of the X-ray source $(B>22.5, R>21$ - Pavlov et al. 2001; $B>23, R>22.5$ - Mereghetti et al. 2002a) rule out the possibility that the X-ray source is an AGN. The lack of variability combined with the X-ray spectral properties makes a cataclysmic variable interpretation also implausible. The nature of the source remains elusive, although an isolated cooling NS or a NS with a "fallback" disk seem to be possible interpretations.

The large frame time, $3.24 \mathrm{~s}$, of the previous snapshot (3 ks) ACIS observation made it impossible to search for short periods and led to strong saturation (pile-up) of the source image, precluding an accurate spectral analysis. To search for pulsations from the compact source and obtain a more accurate spectrum, we observed J0852 with Chandra ACIS with a time resolution of $2.85 \mathrm{~ms}$. We present the results of this observation in $\S 2$ and discuss the nature of the source in $\S 3$. 


\section{Observation and data analysis}

J0852 was observed with ACIS-S3 in Continuous Clocking (CC) mode on 2001 August $30(31.5 \mathrm{ks}$ total exposure). CC mode allows one to achieve time resolution of $2.85 \mathrm{~ms}$ at the expense of spatial information in one dimension. There were no substantial "background flares" during the observation, so we do not exclude any time intervals from the analysis. For data reduction and analysis, we used CIAO 2.2.1 (CALDB 2.7) and XSPEC v.11.0.

The FWHM of the one-dimensional (1-D) source image is 0!.7, consistent with the ACIS point spread function. No evidence for excess emission around the point source is seen above the background of 0.013 counts $\mathrm{s}^{-1}$ per $1^{\prime \prime}$ segment of the 1-D image (equivalent to an average surface brightness of 0.025 counts ks${ }^{-1} \operatorname{arcsec}^{-2}$ ).

\subsection{Spectral Analysis}

For the spectral analysis, we extracted 11,450 source-plus-background counts from a $4^{\prime \prime}$ segment of the 1-D image. The background was taken from two adjacent 10" segments. The background-subtracted source count rate is $0.313 \pm 0.004$ counts $\mathrm{s}^{-1}$. Figure 1 shows the pulse-height spectrum in the $0.6-8.0 \mathrm{keV}$ band, grouped into 77 bins with $\geq 100$ source counts per bin. We ignored all counts below $0.6 \mathrm{keV}$ for spectral fitting because of the poorly known ACIS response at lower energies.

Fitting the spectrum with a power-law (PL) model yields a large photon index $\gamma=$ $4.32 \pm 0.06$ (all uncertainties at a $1 \sigma$ confidence level), and a hydrogen column density $n_{\mathrm{H}, 21} \equiv n_{\mathrm{H}} / 10^{21} \mathrm{~cm}^{-2}=11.2 \pm 0.2$, close to the total Galactic HI column density in this direction, $\approx 1 \times 10^{22} \mathrm{~cm}^{-2}$ (Dickey \& Lockman 1990; estimated with the W3NH tool ${ }^{1}$ ). The quality of the fit is so poor $\left(\chi_{\nu}^{2}=3.94\right.$ for 74 degrees of freedom [d.o.f.] $)$ that this model can be rejected. Thermal plasma emission models (thermal bremsstrahlung and mekal with solar abundances) also do not fit the observed spectrum $\left(\chi_{\nu}^{2}=1.63\right.$ and 14.26 for 74 d.o.f., respectively).

On the contrary, a single blackbody (BB) model fits the spectrum reasonably well $\left(\chi_{\nu}^{2}=1.13\right.$ for 74 d.o.f.; see Fig. 1$)$. It yields a temperature $T=4.68 \pm 0.06 \mathrm{MK}(k T=404 \pm 5$ $\mathrm{eV})$ and a radius of equivalent emitting sphere $R=(0.28 \pm 0.01) d_{1} \mathrm{~km}$, where $d_{1} \equiv d / 1 \mathrm{kpc}$. The bolometric luminosity is $L_{\mathrm{bol}}=(2.5 \pm 0.2) \times 10^{32} d_{1}^{2} \mathrm{ergs} \mathrm{s}^{-1}$. The hydrogen column density, $n_{\mathrm{H}, 21}=3.45 \pm 0.15$, considerably exceeds the highest value, $n_{\mathrm{H}, 21}=0.6$, found

\footnotetext{
${ }^{1}$ http://heasarc.gsfc.nasa.gov
} 
by $\mathrm{Lu} \&$ Aschenbach (2000) for the Vela SNR. It indicates that the source is substantially more distant than the Vela pulsar $\left(d_{\text {Vela }}=294_{-50}^{+76} \mathrm{pc}\right.$ - Caraveo et al. 2001). Adding a PL component to the BB model only marginally improves the fit $\left(\chi_{\nu}^{2}=1.126\right.$ for 72 d.o.f., vs. 1.130 for 74 d.o.f, for a single BB ). The F-test shows that the reduction of $\chi^{2}$ caused by adding the PL component is significant only at a $66 \%$ confidence level.

Fits with the magnetic hydrogen NS atmosphere models (Pavlov et al. 1995) give a lower effective temperature $(k T \approx 270 \mathrm{eV})$ and a larger emitting area $\left(R \approx 1.2 d_{1} \mathrm{~km}\right)$. In both $\mathrm{BB}$ and $\mathrm{H}$ atmosphere fits, the inferred radius is much smaller than the expected NS radius, and the temperature is too high to interpret the detected X-rays as emitted from the whole surface of a uniformly heated isolated NS of a reasonable age.

To constrain the temperature of the entire NS surface, we fit the spectrum with a twocomponent BB model. The fits to the ASCA spectra of the outer, brighter parts of the SNR give a range of hydrogen column densities from 1.4 to $5.3 \times 10^{21} \mathrm{~cm}^{-2}$ (Slane et al. 2001). To find a conservative upper limit on the surface temperature $T_{\mathrm{s}}$, we fix the column density at $n_{\mathrm{H}, 21}=5.3$, add a soft $\mathrm{BB}$ component with $R_{\mathrm{s}}=10 d_{1} \mathrm{~km}$, and fit $T$ and $R$ at different values of $T_{\mathrm{s}}$, increasing $T_{\mathrm{s}}$ until the fit probability falls to $0.1 \%$. This gives an upper limit $T_{\mathrm{s}} \leq 89 \mathrm{eV}$, at a $99.9 \%$ confidence level. If we fix the column density at $n_{\mathrm{H}, 21}=3.4$ (as obtained for the single BB fit), the limit becomes as low as $T_{\mathrm{s}} \leq 75 \mathrm{eV}$.

Although we find no strong spectral lines, there is a hint of a spectral feature at 1.68 $\mathrm{keV}$ (see Fig. 1). This feature persists when the data are rebinned with different numbers of counts per bin. We see no anomalies in the data which could explain the feature as an artifact. In particular, we have ruled out that the feature could be caused by anomalously high values in the bias map for S3 chip (P. Ford 2002, private communication). The shape of the feature resembles the so-called inverse P-Cygni profile, which might be associated with accretion. Its width, $\sim 100 \mathrm{eV}$, might correspond to velocities of accreting material $\sim 0.03 c$; however, this width is comparable with the spectral resolution of ACIS-S3 around $1.7 \mathrm{keV}$ (Chandra Proposers' Observatory Guide, v.3.0, §6.7). The feature is not seen in the background (SNR) spectrum.

\subsection{Timing analysis}

For timing analysis, we extracted 10,957 photons from a 2 ".5 segment centered on J0852 ( $\geq 89 \%$ of these counts are expected to come from the point source). The time span of the observation is $T_{\text {span }}=31.5 \mathrm{ks}$. We corrected the event times for telescope dither and Science Instrument Module motion using the approach described by Zavlin et al. (2000). 
We transformed the corrected times to the solar system barycenter using the axBary tool of CIAO.

We used the $Z_{m}^{2}$ test (Buccheri et al. 1983) to search for periodic pulsations. We calculated $Z_{m}^{2}$ for $m=1-5$ (where $m$ is the number of harmonics included) at $10^{8}$ equally spaced frequencies $f$ in the $0.001-100 \mathrm{~Hz}$ range. This corresponds to oversampling by a factor of about 30 , compared to the expected width of $T_{\text {span }}^{-1} \approx 30 \mu \mathrm{Hz}$ of the $Z_{m}^{2}(f)$ peaks, and guarantees that we miss no peaks. The two most significant peaks we found are at $f=3.324231 \mathrm{~Hz} \pm 3 \mu \mathrm{Hz}(P \approx 301 \mathrm{~ms})$ and $f=30.369484 \mathrm{~Hz} \pm 2 \mu \mathrm{Hz}(P \approx 33 \mathrm{~ms})^{2}$. The most significant $Z_{m, \max }$ values, $Z_{4, \max }=52.9$ for the $301 \mathrm{~ms}$ period and $Z_{1, \max }=36.7$ for the $33 \mathrm{~ms}$ period, correspond to $96.7 \%$ and $96.8 \%$ significance levels, respectively, for the number of independent trials $\mathcal{N}=f_{\text {max }} T_{\text {span }} \approx 3 \times 10^{6}$.

The pulsed fractions obtained from the pulse profiles are $13 \% \pm 3 \%$ and $9.1 \% \pm 2.5 \%$ for the $301 \mathrm{~ms}$ and $33 \mathrm{~ms}$ period candidates, respectively. Because of the low significance, we consider $13 \%$ as an upper limit for the pulsed fraction.

To search for variability on larger time scales, we binned the data into $200 \mathrm{~s}$ bins. Using the Kolmogorov-Smirnov test, the hypothesis that the observed numbers of counts in the bins come from a Poisson distribution (with the mean of 69.756 counts per bin) can not be rejected at a $70 \%$ confidence level. We have also used the Fourier transform and found no periodic signal with a pulsed fraction larger than $12 \%$ in $1-10 \mathrm{mHz}$ frequency range. Therefore, we find no evidence for long-term variability in the data.

\section{Discussion}

The X-ray data and optical limits indicate that J0852 is the compact remnant (NS or $\mathrm{BH}$ ) of the supernova explosion. The X-ray spectral properties and the lack of radio emission (Duncan \& Green 2000) suggest that J0852 is not an active pulsar. Furthermore, the Chandra observations show no sign of a pulsar-wind nebula $(\mathrm{PWN})$ around the point source. From the $3 \mathrm{ks}$ observation in Timed Exposure mode (Pavlov et al. 2001), the $3 \sigma$ upper limit on the PWN brightness (in counts $\operatorname{arcsec}^{-2}$ ) can be estimated as $3(b / A)^{1 / 2}$, where $b=0.029$ counts $\operatorname{arcsec}^{-2}$ is the background surface brightness, and $A$ is the (unknown) PWN area. Scaling the area as $A=1000 A_{3} \operatorname{arcsec}^{2}$ (which corresponds to the transverse size of about $5 \times 10^{17} A_{3}^{1 / 2} \mathrm{~cm}$ ) and assuming a PL spectrum with a photon index $\gamma=1.5-2$, we obtain

\footnotetext{
${ }^{2}$ The frequency uncertainties, at a 90\% confidence level, are estimated using the method of Gregory \& Loredo (1996); see also Zavlin et al. (2000).
} 
an upper limit of $(1.3-2.0) \times 10^{30} A_{3}^{1 / 2} d_{1}^{2} \mathrm{erg} \mathrm{s}^{-1}$ on the PWN luminosity in the $0.2-10 \mathrm{kev}$ band, for $n_{\mathrm{H}, 21}$ in the range of 1.4-5.3.

The observational properties of J0852 strongly resemble those of the other radio-quiet central compact objects (CCOs) in SNRs (see Pavlov et al. 2002a for a review), particularly the CCO in the SNR Cas A (Murray et al. 2002, and references therein). At least one of these sources, 1E 1207.4-5209, has been proven to be a NS rotating with a period of $424 \mathrm{~ms}$ (Zavlin et al. 2000; Pavlov et 2002b). A number of possible interpretations of CCOs have been recently discussed by several authors (e.g. Pavlov et al. 2000, 2001, 2002a; Chakrabarty et al. 2001). The limits on X-ray-to-optical flux ratio for J0852 and the Cas A CCO virtually rule out models which involve accretion onto a NS or a BH from a binary companion. If these are accreting objects, a more plausible source of accreting matter might be a "fossil disk", left over after the SN explosion (van Paradjis, Taam, \& van den Heuvel 1995). Alternatively, thermal emission from an isolated, cooling NS could explain the observational results. We discuss these two options below.

\subsection{Accretion-powered X-ray pulsar?}

If J0852 is an accreting NS, the observed luminosity, $L_{\mathrm{x}} \sim 2 \times 10^{32} d_{1}^{2} \mathrm{erg} \mathrm{s}^{-1}$, could be due to a rather low accretion rate, $\dot{m} \sim 1.5 \times 10^{12} R_{6} M_{1}^{-1} d_{1}^{2} \mathrm{~g} \mathrm{~s}^{-1}$, where $R_{6}=R_{\mathrm{NS}} /\left(10^{6} \mathrm{~cm}\right)$, $M_{1}=M / M_{\odot}$. The accreting matter could be supplied from a fossil ("fallback") disk. The formation of such a disk from the ejecta produced by a SN explosion was discussed by a number of authors (e.g. Marsden, Lingenfelter, \& Rothschild 2001, and references therein). Some models suggest that a fossil disk can be formed several days after the SN explosion ("prompt" disk) and range from $0.001 M_{\odot}$ to $0.1 M_{\odot}$, while others suggest that the disk can be formed later, years after the SN explosion ("delayed" disk). The details of the formation mechanism and the disk properties are highly uncertain, and, consequently, the accretion rate $\dot{m}$ is also poorly constrained, but the required value of $\sim 10^{12} \mathrm{~g} \mathrm{~s}^{-1}$ is low enough not to exhaust the disk at any reasonable age of J0852.

The accretion onto a NS can proceed in two different regimes (e.g., Frank, King, \& Raine 1992), depending on the relation between the corotation radius, $R_{c}=1.5 \times 10^{8} P^{2 / 3} M_{1}^{1 / 3} \mathrm{~cm}$, and the magnetospheric radius, $R_{M}=3.5 \times 10^{9} B_{12}^{4 / 7} \dot{m}_{12}^{-2 / 7} M_{1}^{-1 / 7} R_{6}^{12 / 7} \mathrm{~cm}$, where $P$ is the NS spin period, $B=10^{12} B_{12} \mathrm{G}$ is the magnetic field at the NS surface, and $\dot{m}_{12}=\dot{m} /\left(10^{12} \mathrm{~g} \mathrm{~s}^{-1}\right)$. If $R_{M}>R_{c}$, the infalling material is stopped at the magnetospheric radius and expelled as a wind due to centrifugal force. In this "propeller regime" (Illarionov \& Sunyaev 1975), X-ray emission is mainly due to optically thin thermal bremsstrahlung produced in the flow (Wang \& Robertson 1985). Since the thermal bremsstrahlung model does not fit the observed 
spectrum, we consider this case unlikely.

$$
\text { If } R_{M}<R_{c} \text { (i.e., } P \gtrsim 10^{2} B_{12}^{6 / 7} \dot{m}_{12}^{-3 / 7} M_{1}^{-5 / 7} R_{6}^{18 / 7} \text { s, or } B \lesssim 4 \times 10^{9} P^{7 / 6} \dot{m}_{12}^{1 / 2} M_{1}^{5 / 6} R_{6}^{-3}
$$
$\mathrm{G})$, the accreting matter is able to reach the NS surface. At extremely low magnetic fields, $B \lesssim 6 \times 10^{5} \dot{m}^{1 / 2} M_{1}^{1 / 4} R_{6}^{-5 / 4} \mathrm{G}$, when the magnetospheric radius is smaller than the NS radius, a hot layer is formed at the boundary between the accretion disk and the NS surface (e.g., Frank et al. 1992). Since this boundary layer is expected to be optically thin at $\dot{m} \ll 10^{16} \mathrm{~g} \mathrm{~s}^{-1}$ (Inogamov \& Sunyaev 1999), its radiation cannot explain the observed $\mathrm{BB}$ spectrum. At reasonable magnetic fields, $B \gg 10^{6} \dot{m}^{1 / 2} M_{1}^{1 / 4} R_{6}^{-5 / 4} \mathrm{G}\left(R_{M} \gg R_{\mathrm{NS}}\right)$, the accretion flow is channeled onto the NS poles, producing hot spots of radius $a \sim R_{\mathrm{NS}}^{3 / 2} / R_{M}^{1 / 2} \sim$ $0.17 B_{12}^{-2 / 7} \dot{m}_{12}^{1 / 7} M_{1}^{1 / 14} R_{6}^{9 / 14} \mathrm{~km}$. The observed size and temperature of the BB-like radiation are consistent with being emitted from such a cap at $B \sim 10^{11} \mathrm{G}$. Such an estimate requires a pulsar period $P \gtrsim 10 \mathrm{~s}$, much longer than our candidate periods. If we assume $P=301$ ms, the condition $R_{M}<R_{c}$ requires $B \lesssim 10^{9} \dot{m}_{12}^{1 / 2} M_{1}^{5 / 6} R_{6}^{-3} \mathrm{G}$ and $a \gtrsim 1.2 \dot{m}_{12}^{1 / 7} M_{1}^{1 / 14} R_{6}^{9 / 14}$ $\mathrm{km}$, considerably larger than the size of emitting region, $R \approx 0.3 d_{1} \mathrm{~km}$, inferred from the BB fit. However, given the crudeness of the polar cap size estimate, which can be much smaller than adopted above (see, e.g., Frank et al. 1992, and references therein), we cannot rule out the candidate period of $301 \mathrm{~ms}$ based on the apparent inconsistency between $a$ and $R$. Thus, in the accretion hypothesis, J0852 could be a low-luminosity X-ray pulsar, presumably with a magnetic field much lower than those of binary X-ray pulsars, slowly accreting from a fossil disk. An argument against this interpretation is a lack of nonperiodic variability in the radiation from J0852, which is commonly observed from accreting sources (at least, $\mathrm{X}$-ray binaries). On the other hand, variability could be found in further observations of this source. A direct confirmation of the accreting hypothesis would be detection of an accretion disk, which would require deep IR-optical observations with high angular resolution.

\subsection{Isolated cooling neutron star?}

One can also assume that J0852 is an isolated (non-accreting) NS emitting thermal radiation from its surface. The "standard" NS cooling models predict a luminosity of $\sim(0.5-$ 2) $\times 10^{34} \mathrm{erg} \mathrm{s}^{-1}$ for a NS of 0.1-10 kyr age (e.g., Tsuruta 1998). The lower observed luminosity of J0852 could be interpreted as due to an accelerated cooling mechanism, but applicability of the cooling models to J0852 is questionable because the models assume a uniformly heated NS surface while the size of the emitting region obtained from the BB fit is only $\approx 0.3 d_{1} \mathrm{~km}$.

Apparent sizes of the emitting regions much smaller than the canonical NS radius have been observed from other isolated NSs (Pavlov et al. 2002a,c). In particular, the Cas A 
CCO shows a (blackbody) size of $0.3 \mathrm{~km}$ with a temperature of $0.6 \mathrm{keV}$ (Pavlov et al. 2000), which hints that it is an object similar to J0852, with a higher temperature possibly due to its younger age. Pavlov et al. (2000) suggested a two-component thermal model for the Cas A CCO, in which the observed X-rays are emitted from hydrogen polar caps of about $1 \mathrm{~km}$ radius and $0.24 \mathrm{keV}$ effective temperature, while the rest of the NS surface is iron at a temperature of $0.15 \mathrm{keV}$, too cold to be observable because of strong interstellar absorption. In this model, the polar caps are hotter because of the higher thermal conductivity of hydrogen. Weaker ISM absorption for J0852 allowed us to find a lower temperature limit for the cold component, $<90 \mathrm{eV}$, too low to explain the temperature difference by different chemical compositions. It should be mentioned that this limit is a factor of 1.4 lower than the temperature predicted by the so-called standard (slow) cooling model for a $10^{3}$ yrs old NS (see, e.g., Fig. 4 in Slane, Helfand, \& Murray 2002). This may indicate that if J0852 is a NS, it undergoes fast cooling, perhaps associated with direct Urca processes in the NS core (e.g., Yakovlev et al. 2002).

Hot spots on the NS surface could also be associated with a very strong magnetic field, $B \gg 10^{13}$ G. Due to anisotropic heat conductivity of the NS crust, the surface temperature is higher at the magnetic poles (Greenstein \& Hartke 1983; Shibanov \& Yakovlev 1996). To produce small hot spots, the surface magnetic field should be strongly nonuniform (e.g., an offset dipole or a quadrupole - Page \& Sarmiento 1996). Fast decay of a superstrong magnetic field $\left(B \gtrsim 10^{14} \mathrm{G}\right.$ ) could provide an additional source of polar cap heating (Thompson \& Duncan 1996; Colpi, Geppert \& Page 2000). In such strong magnetic fields, electron-positron pair creation should be suppressed due to photon splitting (Baring \& Harding 2001), which is consistent with the apparent lack of pulsar activity in J0852.

One can crudely estimate the magnetic field assuming that one of the two candidate periods, $33 \mathrm{~ms}$ or $301 \mathrm{~ms}$, is the true period. If the initial period of the pulsar was much shorter than the current period, then the period derivative, rotation energy loss rate, and "canonical" magnetic field $\left[B \equiv 3.2 \times 10^{19}(P \dot{P})^{1 / 2} \mathrm{G}\right]$, can be estimated as $\dot{P}=3.2 \times$ $10^{-11} P\left[(n-1) t_{3}\right]^{-1}, \dot{E}=1.25 \times 10^{36} P^{-2}\left[(n-1) t_{3}\right]^{-1} \mathrm{erg} \mathrm{s}^{-1}$, and $B=1.8 \times 10^{14} P\left[(n-1) t_{3}\right]^{-1 / 2}$ $\mathrm{G}$, where $t=10^{3} t_{3}$ yr is the NS age, and $n$ is the braking index. Assuming $n=2.5$, (close to that observed in young pulsars), we obtain, for $P=33 \mathrm{~ms}, \dot{P}=7.0 \times 10^{-13} t_{3}^{-1}$, $\dot{E}=8.6 \times 10^{38} t_{3}^{-1} \mathrm{erg} \mathrm{s}^{-1}$, and $B=4.9 \times 10^{12} t_{3}^{-1 / 2} \mathrm{G}$ - parameters typical for a young, active pulsar, in apparent contradiction with observations. On the other hand, for the 301 ms period, we obtain $\dot{P}=6.4 \times 10^{-12} t_{3}^{-1}, \dot{E}=9.2 \times 10^{36} t_{3}^{-1} \mathrm{erg} \mathrm{s}^{-1}$, and $B=4.4 \times 10^{13} t_{3}^{-1 / 2}$ G. Since the local magnetic field can be much higher than the canonical value (e.g., for an offset dipole), one can speculate that, for $P=301 \mathrm{~ms}$, it is high enough to explain the hot $\operatorname{spot}(\mathrm{s})$ and the lack of radio-pulsar activity. If this hypothesis is correct, the J0852 could be a very young Anomalous X-ray Pulsar (AXP) whose period will become of order 6-12 s (as 
observed in AXPs) when it grows older by a factor of 20-40.

However, there are considerable differences between the properties of AXPs and J0852. Contrary to AXPs, whose spectra contain both the BB and PL components of comparable luminosities (Mereghetti et al. 2002b), the spectrum of J0852 fits well with a single BB model. The size of the emitting region in J0852 is substantially smaller $(0.3 \mathrm{~km}$ vs. $0.7-5$ $\mathrm{km}$ ), and the temperature somewhat lower $(0.4 \mathrm{keV}$ vs. $0.4-0.6 \mathrm{keV})$, than those of AXPs. These differences (particularly, the lack of a PL component in J0852) hint at different NS parameters. For instance, it is quite possible that none of the candidate periods is correct, and the true period is even longer than the AXP periods. In this case, the magnetic field could be even higher than those adopted in the magnetar interpretation of AXPs - e.g., $B=2.4 \times 10^{15}(P / 20 \mathrm{~s}) t_{3}^{-1} \mathrm{G}$. Such a strong field can inhibit not only the pair cascade, but also the emission of primary particles from the NS surface, which might explain the lack of particles in the NS magnetosphere (hence, the lack of nonthermal radiation) in J0852. If the NS rotates sufficiently slow, $P \gtrsim 0.5 B_{15}^{4 / 15}(Z / 26)^{-12 / 15} \mathrm{~s}$, the critical parallel electric field required to pull out electrons from the NS surface, $E_{\|, \text {crit }} \approx 2.7 \times 10^{12}(Z / 26)^{6 / 5} B_{15}^{3 / 5} \mathrm{~V} \mathrm{~cm}^{-1}$ (Usov \& Melrose 1995), is higher than the maximum parallel electric field at the surface, $E_{\|, \max } \approx 1 \times 10^{10} B_{15}(P / 20 \mathrm{~s})^{-3 / 2} \mathrm{~V} \mathrm{~cm}^{-1}$. On the other hand, the surface temperature, $k T_{e} \approx 0.5(Z / 26)^{4 / 5} B_{15}^{2 / 5} \mathrm{keV}$, above which the thermoionic emission of electrons becomes efficient (Usov \& Melrose 1995), grows with increasing magnetic field. (These estimates assume that the NS has no light-element [e.g., hydrogen] atmosphere.) Therefore, a long period and a superstrong magnetic field might explain the lack of the PL tail in the spectrum of J0852 and other enigmatic CCOs (e.g., in the Cas A and Pup A SNRs; Pavlov et al. 2002a).

In summary, the observations of J0852 can be explained assuming it is a NS. Given the deep limiting optical magnitudes and the lack of nonperiodic variability, we consider the interpretation in terms of an isolated NS with a very strong magnetic field somewhat more plausible than the accretion models. Further observations are required to confirm or reject this hypothesis. Particularly important would be X-ray timing observations to measure the period unequivocally, high-resolution X-ray spectral observations to look for spectral features, and IR-optical observations to search for a NS counterpart (e.g., a fossil disk).

We thank Glenn Allen and Allyn Tennant for the advice on the ACIS timing issues, Leisa Townsley and George Chartas for the advice on the spectral issues, Peter Ford for examining the bias map, Bing Zhang for useful discussions, and the anonymous referee for valuable comments. This work was partly supported by NASA grants NAG5-10865, NAS838252, and NAS8-01128. 


\section{REFERENCES}

Aschenbach, B. 1998, Nature, 396, 141

Aschenbach, B., Iyudin, A. F., \& Schönfelder, V. 1999, A\&A, 350, 997

Baring, M. G., \& Harding, A. K. 2001, ApJ, 547, 929

Buccheri, B., et al. 1983, A\&A, 128, 245

Caraveo, P. A., De Luca, A., Mignani, R. P., \& Bignami, G. F. 2001, ApJ, 561, 930

Chakrabarty, D., Pivovaroff, M. J., Hernquist, L. E., Heyl, J. S., \& Narayan, R. 2001, ApJ, 548,800

Colpi M., Geppert U., \& Page D. 2000, ApJ, 529, L29

Dickey, J. M., \& Lockman, F. J. 1990, ARA\&A, 28, 215

Duncan, A. R., \& Green, D. A. 2000, A\&A, 364, 732

Frank, J., King, A., \& Raine, D. 1992, Accretion Power in Astrophysics (Cambridge: Cambridge Univ. Press)

Greenstein, G., \& Hartke, G. J. 1983, ApJ, 271, 283

Gregory, P. C., \& Loredo, T. J. 1996, ApJ, 473, 1059

Illarionov, A. F., \& Sunyaev, R. A. 1975, A\&A, 39, 185

Inogamov, N. A., \& Sunyaev, R. A. 1999, Astron. Lett., 25, 269

Iyudin, A. F., et al. 1998, Nature, 396, 142

Lu, F. J., \& Aschenbach, B. 2000, A\&A, 362, 1083

Marsden, D., Lingenfelter, R. E., \& Rothschild, R. E. 2001, ApJ, 547, L45

Mereghetti, S. 2001, ApJ, 548, L213

Mereghetti, S., Pelizzoni, A., \& De Luca, A. 2002a, in Neutron Stars in Supernova Remnants, ASP Conf. Ser., v.271, eds. P. O. Slane \& B. M. Gaensler (San Francisco: ASP), 289

Mereghetti, S., Chiarlone, L., Israel, G. L., \& Stella, L. 2002b, in Proc. 270 Heraeus Seminar on Neutron Stars, Pulsars and Supernova Remnants, eds. W. Becker, H. Lesch, \& J. Trümper, MPE Reports, in press (astro-ph/0205122) 
Murray, S. S., Ransom, S. M., Juda, M., Hwang, U., \& Holt, S. S. 2002, ApJ, 566, 1039

Page, D., \& Sarmiento, A. 1996, ApJ, 473, 1067

Pavlov, G. G., Shibanov, Yu. A., Zavlin, V. E., \& Meyer, R. D. 1995, in The Lives of the Neutron Stars, eds. M. A. Alpar, Ü. Kiziloglu, \& J. van Paradijs (Dordrecht: Kluwer), 71

Pavlov, G. G., Zavlin, V. E., Aschenbach, B., Trumper, J., \& Sanwal, D. 2000, ApJ, 531, L53

Pavlov, G. G., Sanwal, D., Kiziltan, B., \& Garmire G. P. 2001 ApJ, 559, L131

Pavlov, G. G., Sanwal, D., Garmire G. P., \& Zavlin, V. E. 2002a, in Neutron Stars in Supernova Remnants, ASP Conf. Ser., v.271, eds. P. O. Slane \& B. M. Gaensler (ASP: San Francisco), 247

Pavlov, G. G., Zavlin, V. E., Sanwal, D., \& Trümper, J. 2002b, ApJ, 569, L95

Pavlov, G. G., Zavlin, V. E., \& Sanwal, D. 2002c, in Proc. 270 Heraeus Seminar on Neutron Stars, Pulsars and Supernova Remnants, eds. W. Becker, H. Lesch, \& J. Trümper, MPE Reports, in press (astro-ph/0206025)

Shibanov, Y. A., \& Yakovlev, D. G. 1996, A\&A, 309, 171

Slane, P., Hughes, J. P., Edgar, R. J., Plucinsky, P. P., Miyata, E., Tsunemi, H., \& Aschenbach, B. 2001, ApJ, 548, 814

Slane, P. O., Helfand, D. J., \& Murray, S. S. 2002, ApJ, 571, L45

Thompson, C., \& Duncan, R. C. 1996, ApJ, 473, 322

Tsuruta, S., 1998, Phys. Rep., 292, 1

Tsunemi, H. et al. 2000, PASJ, 52, 887

Usov, V. V., \& Melrose, D. B. 1995, Australian Journ. Phys., 48, 571

van Paradijs, J., Taam, R. E., \& van den Heuvel, F. P. G. 1995, A\&A, 299, L41

Wang, Y.-M., \& Robertson, J. A. 1985, A\&A, 151, 361

Yakovlev, D. G., Gnedin, O. Y., D. G., Kaminker, \& Potekhin, A. Y. 2002, in Proc. 270. Heraeus Seminar on Neutron Stars, Pulsars and Supernova Remnants, eds. W. Becker, H. Lesch, \& J. Trümper, MPE Reports, in press (astro-ph/0204226) 
Zavlin, V. E., Pavlov, G. G., Sanwal, D., \& Trum̈per, J. 2000, ApJ, 540, L25 


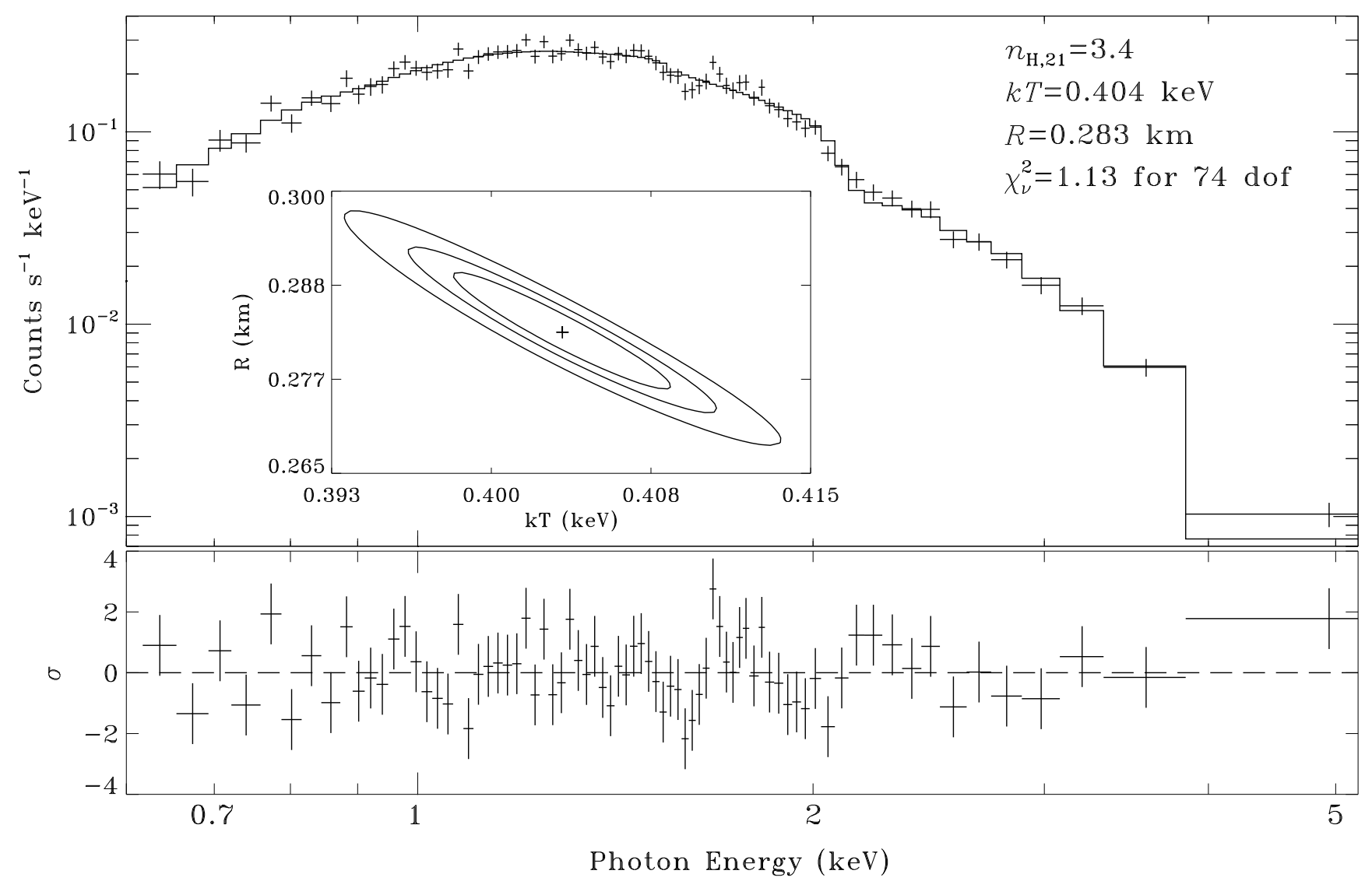

Fig. 1.- Fit of the ACIS-S3 spectrum of J0852 with a blackbody model. The contours correspond to $68 \%, 90 \%$ and $95 \%$ confidence levels. A possible spectral feature is seen at about $1.68 \mathrm{keV}$. 